\title{
Comparaison entre les rendements industriels et modèle pour le fonctionnement en turbinage inversé des groupes bulbes de Cambeyrac et Saint-Malo
}

\author{
Comparison between prototype and model efficiencies \\ achieved with the Cambeyrac and Saint-Malo bulb units \\ when running as reverse-flow turbines
}

\author{
PAR J. CHEVALIER
}

\author{
INGÉNIEUR A LA DIRECTION DES ÉTUDES ET RECHERCHES D'ÉLECTRICITÉ DE FRANGE \\ CHEF DE LA DIVISION «STATION D'ESSAIS DE TURBINES》 \\ AU SERVICE DES LABORATOIRES ET ESSAIS HYDRAULIQUES, CHATOU
}

\begin{abstract}
Contróles de similitude géométrique et hydraulique effectués sur le groupe bulbe de Cambeyrac (groupe $N$ ) et sur son modèle réduit de diamiètre $530 \mathrm{~mm}$.

Méthode de transposition du rendement modèle au rendement industriel, utilisant des paramètres obtenus grâce aux essais industriels. Application à Cambeyrac: résultats satisfaisants $\dot{a}$ moins de $1 \%$ près.

Comparaison des essais industriels de l'aménagement de Saint-Malo aux résultats obtenus sur le modèle de diamètre $500 \mathrm{~mm}$, permettant de déterminer les mêmes paramètres de transposition et d'extrapoler avec sécurité la zone de fonctionnement Saint-Malo en turbinage inversé à la zone de fonctionnement Rance pour la même machine.
\end{abstract}

Dans la communication précédente, où sont traités les essais de rendements effectués sur le groupe bulbe industriel de Saint-Malo, M. DUBors a exposé les méthodes utilisées et les résultats des mesures. On peut se rendre compte, notamment en ce qui concerne le fonctionne-

\begin{abstract}
Geometrical and hydraulic similitude checks carried out with the Cambeyrac bulb unit (Unit $N$ ) and its $530 \mathrm{~mm}$ diameter scale model. The method used to transpose the model efficiency to the prototype efficiency with the aid of parameters obtained from the prototype tests. The application of the method to Cambeyrac produced results agreeing to within less than $1 \%$.

By comparing the results of tests on the SaintMalo prototype and on its $500 \mathrm{~mm}$ dia. moder, the same transposition parameters could be determined and the reverseflow turbine operation zone at Saint-Malo reliably extrapolated to the Rance operating zone for the same machine.
\end{abstract}

ment en turbinage inversé, que la zone qu'il a été possible de couvrir par des essais industriels est réduite par rapport à la zone de fonctionnement normale de la machine dans le cas où elle aurait été installée à la Rance.

Dans le but de chiffrer l'effet d'échelle à atten- 
dre au cour de la colline, nous avons été amenés à mettre au point une méthode de comparaison entre le modèle et le prototype à partir d'essais complets effectués notamment à Cambeyrac. La justification des mesures effectuées et de la méthode employée fait l'objet de la première partie de cet exposé.

La seconde partie a trait à l'aménagement de Saint-Malo. Les conditions d'implantation de ce groupe industriel marémoteur type «Rance» ne permettent son utilisation que dans une zone de chutes réduites. Cependant, grâce aux relevés complets effectués sur modèle et à la méthode exposée relativement à Cambeyrac, il a été possible de prédire les performances à attendre de cette machine sous les chutes de la Rance.

Dans cet exposé nous nous bornerons à l'étude des fonctionnements dits à «distributeur fixe », soit en turbine, soit en pompe, c'està-dire à des fonctionnements pour lesquels le distribuleur a une position fixe (généralement tout ouvert), quel que soit le point de fonctionnement de Ia machine. Le régulateur agit alors directement sur les pales de la roue, seul paramètre de réglage.

\section{I. - ESSAIS CAMBEYRAC}

C'est sur le fonctionnement en turbinage inversé de la machine Neyrpic de Cambeyrac, que s'est porłé plus particulièrement notre effort en ce qui concerne la vérification de la similitude entre les fonctionnements sur modèle et industriel.

En effet, la machine industrielle de Cambeyrac peut être alimentée en turbine inversée (c'est-à-dire l'eau atteignant la roue avant le distributeur) par l'artifice d'un canal latéral mettant directement en communication le plan d'eau amont de la retenue et la sortie normale de la turbine. L'effet perturbateur dû à la présence des deux coudes à $90^{\circ}$ dans l'alimentation de la machine est compensé par l'installation de guideau. Il était impossible de représenter sur le modèle un canal latéral semblable à celui-ci. On a donc cherché à relever sur place la répartition des vitesses à l'entrée de la machine et à la reproduire à l'entrée du modèle.

La comparaison des courbes de répartition de vitesses obtenues montre que leur correspondance est suffisamment bonne pour que l'on puisse considérer que le modèle et la machine réelle sont alimentés dans les conditions semblables.

La similitude géométrique du conduit hydraulique du modèle de $530 \mathrm{~mm}$ de diamètre de roue a été contrôlée point par point depuis l'entrée de la turbine jusqu'à la sortie, définies par les extrémités d'aspirateur. Notamment les profils de bulbe, de ceinture et de directrices ont été soigneusement relevés, ainsi que les diamètres des principales parties en place. Le profil des pales lui-même a pu être contrôlé de très près grâce à une méthode de photogrammétrie et de restitution comparant la pale modèle à la pale industrielle.

Pratiquement, et après quelques retouches effectuées sur le modèle réduit, nous ne pensons pas qu'il subsiste d'erreur de similitude pouvant entacher la comparaison que nous avons en vue.
Du point de vue des mesures, précisons que la puissance industrielle sur l'arbre a été calculée en retirant de la puissance mesurée aux bornes de l'alternateur, non seulement les pertes relatives à celui-ci, mais encore les pertes de palier et de butée. Celles-ci sont normalement imputables à la turbine, mais n'existent pas sur le modèle, dont tous les frottements sont pesés par un système de mise en balance de l'ensemble en rotation.

La chute industrielle et la chute modèle ont été définies de la même manière comme différence des lignes de charge amont et aval des sections de mesure semblables. Le repérage des ouvertures du distributeur et des inclinaisons de pales a été réalisé de manière géométrique (mesures d'angles), et des relevés sur la machîne industrielle ont permis de s'assurer que le repérage utilisé sur place (course de servo-moteur) conduisait à une définition géométrique de l'inclinaison identique à celle utilisée sur modèle.

Nous avons insisté sur ces conditions de similitude, car elles sont particulièrement critiques dans une telle installation, ou la chute d'essais est essentiellement faible et variable (de 0 à 10 mètres) et où les pertes de charge dans les ouvrages d'amenée ainsi que les hauteurs dynamiques d'entrée et de sortie peuvent prendre des valeurs relatives importantes.

Les essais industriels effectués ont permis, pour un certain nombre de points de fonctionnement à ouverture de pales constante et à chute variable, de tracer en particulier les courbes de rendement, de puissance sur l'arbre et de débit en fonction de la hauteur de chute nette. Les résultats modèle ont été présentés de facon analogue.

Les résultats d'essais se présentent donc pour le modèle et pour le prototype par un ensemble de courbes travées à vitesse constante et à distributeur fixe, l'inclinaison $i$ des pales étant prise comme paramètre et la chute d'essais, comme variable : 


$$
\begin{aligned}
& \left.\begin{array}{l}
\mathrm{Q}=f(h) \\
\mathrm{W}=g(h) \\
\eta=h(h)
\end{array}\right\} \text { résultat modèle } \\
& \left.\begin{array}{r}
Q^{\prime}=f^{\prime}(h) \\
W^{\prime}=g^{\prime}(h) \\
\eta^{\prime}=g^{\prime}(h)
\end{array}\right\} \text { résultats industriels }
\end{aligned}
$$

Dans l'ensemble des résultats obtenus, on peut remarquer que les courbes

$$
\mathrm{Q}=f(\mathrm{H}) \quad \text { et } \mathrm{Q}^{\prime}=f^{\prime}(\mathrm{H})
$$

sont des paraboles très tendues et sensiblement parallèles entre elles pour une même inclinaison de pales, et que les courbes

$$
\mathrm{W}=g(\mathrm{H}) \quad \text { et } \mathrm{W}^{\prime}=g^{\prime}(\mathrm{H})
$$

sont assimilables à des droites, à une très faible approximation près, dans toute la zone des fonctionnements de la machine.

On peut ainsi écrire que, pour une inclinaison de pales déterminée $i$ le point de fonctionnement de paramètres modèle $\mathrm{Q}, \mathrm{W}, \mathrm{H}, \eta$ se transforme en un point de paramètres réels $Q^{\prime}, W^{\prime}, \mathrm{H}^{\prime}$, $\eta$ et les relations suivantes entre ces paramètres s'écrivent :

$$
\begin{aligned}
\mathrm{Q} & =f(\mathrm{H}) \\
\mathrm{W} & =\mathrm{K}\left(\mathrm{H}-\mathrm{H}_{0}\right) \\
\mathrm{H}_{0} & =\text { 《Chute minimale turbinale } » \\
\eta & =\frac{\mathrm{W}}{\bar{\omega} \mathrm{QH}} \\
\mathrm{Q}^{\prime}-\mathrm{Q}_{0}=\mathrm{Q}=f(\mathrm{H}) & \mathrm{W}^{\prime}=\mathrm{K}^{\prime}\left(\mathrm{H}-\mathrm{H}_{0}^{\prime}\right) \\
\eta^{\prime} & =\frac{\mathrm{W}^{\prime}}{\bar{\omega}^{\prime} \mathrm{Q}^{\prime} \mathrm{H}}
\end{aligned}
$$

c'est-à-dire :

$$
\eta=\frac{\mathrm{K}\left(\mathrm{H}-\mathrm{H}_{0}\right)}{\bar{\omega} \mathrm{QH}}
$$

et

$$
\eta^{\prime}=\frac{\mathbf{K}^{\prime}\left(\mathrm{H}-\mathbf{H}_{0}\right)}{\bar{\omega}^{\prime}\left(\mathrm{Q}+\mathrm{Q}_{0}\right) \mathbf{H}}
$$

soit

$$
\frac{\eta^{\prime}}{\eta}=\frac{\mathrm{K}^{\prime}}{\mathrm{K}} \frac{\omega}{\omega^{\prime}} \frac{\mathrm{Q}}{\mathrm{Q}+\mathrm{Q}_{0}} \frac{\mathrm{H}-\mathrm{H}_{0}^{\prime}}{\mathrm{H}-\mathrm{H}_{0}}
$$

comme, dans tous nos relevés

$$
\mathrm{Q}_{0} \leqslant 3 \% \mathrm{Q} \quad \frac{1}{1+\left(\mathrm{Q}_{0} / \mathrm{Q}\right)} \# 1-\frac{\mathrm{Q}_{0}}{\mathrm{Q}}
$$

par suite

$$
\frac{\eta^{\prime}}{\eta}=\frac{\mathrm{K}^{\prime}}{\mathrm{K}} \frac{\omega}{\omega^{\prime}}\left(1-\mathrm{Q}_{0} / \mathrm{Q}\right)\left[1+\frac{\mathrm{H}_{0}-\mathrm{H}_{0}^{\prime}}{\mathrm{H}-\mathrm{H}_{0}}\right]
$$

Dans cette relation, interviennent les paramètres suivants :

- rapport des pentes des droites puissancehauteur;

- décalage en débit des courbes débit-hauteur;

- décalage des chutes minimales turbinables.

Chacun de ces paramètres doit pouvoir être défini pour chacune des inclinaisons de pales réalisées. Les essais modèle et industriels permettent ainsi de tracer les courbes

$$
\begin{array}{rlrl}
\mathrm{K} & =u(i) & \mathrm{K}^{\prime} & =u^{\prime}(i) \\
\mathrm{H}_{0} & =v(i) & \mathrm{H}_{0}^{\prime} & =v^{\prime}(i) \\
\mathrm{Q}_{0} & =w(i) &
\end{array}
$$

dont les graphiques relatifs à Cambeyrac sont joints en annexe. (fig. 1, 2, 3).

A partir de celles-ci, le calcul permet de passer directement de la colline modèle à la colline industrielle.

Deux exemples d'application sont donnés dans les graphiques joints, où, pour le fonctionnement en turbine inversée de la machine de Cambeyrac, on a représenté en fonction de la chute

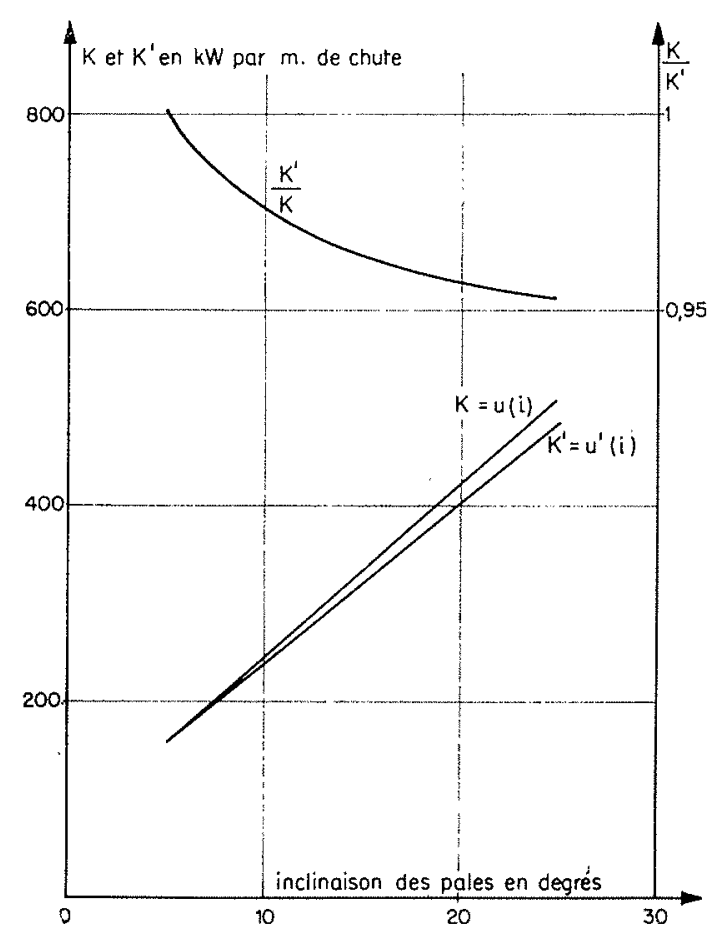

FIG. 1

Cambeyrac. Turbinage inversé. Pente des droites puissance-hauteur. 


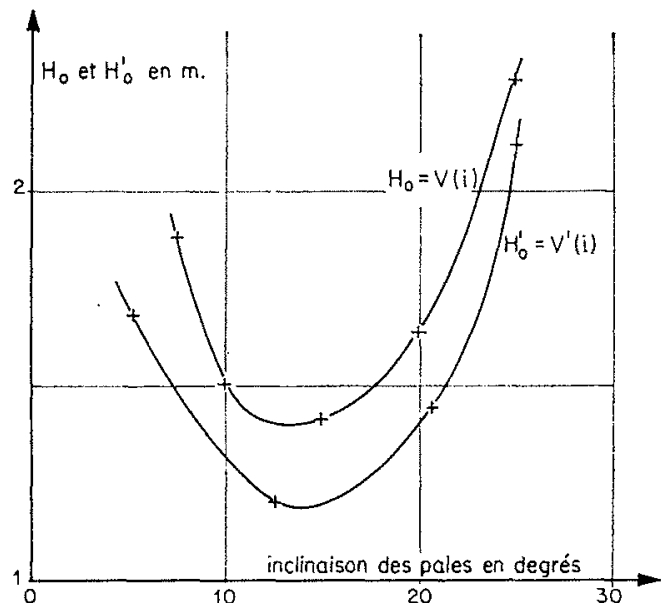

FIG. 2

Cambeyrac. Turbinage inversé. Chute minimales turbinables.

les courbes du rendement modèle, du rendement industriel calculé par ce procédé et les points bruts d'essais industriels (fig. 4, 5).

On voit que, dans tous les cas, les écarts maximaux entre la courbe industrielle calculée et les points d'essais n'excèdent pas $1 \%$, cet écart pouvant d'ailleurs être imputé à la dispersion des mesures.

Cette formule n'est en aucun cas une formule de transposition, puisqu'il faut avoir fait suffsamment d'essais modèle, et surtout industriels, pour pouvoir en obtenir les différents paramètres et qu'aussi bien, ces essais étant faits, il

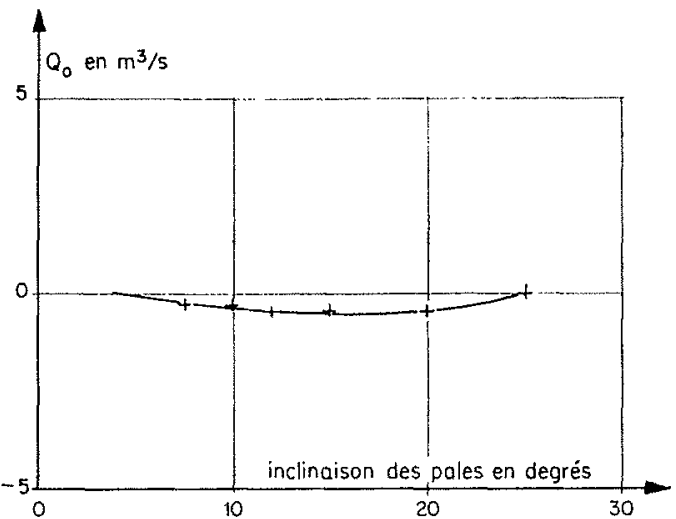

FIG. 3

Cambeyrac. Turbinage inversé. Décalage des courbes débit-hauteur.

serait possible sans plus de tracer la colline industrielle.

Elle offre cependant l'avantage de permettre un tracé de la courbe industrielle basé sur la forme de la courbe modèle et restant précis même dans une zone où les essais seraient peu nombreux. Elle moyenne l'ensemble des essais industriels et rend plus homogène le résultat final obtenu.

En outre, et c'est là son avantage principal, elle permet le tracé de la courbe industrielle avec une boune précision dans une zone où les essais pourraient éventuellement ne pas avoir été effectués, comme c'est le cas pour Saint-Malo.

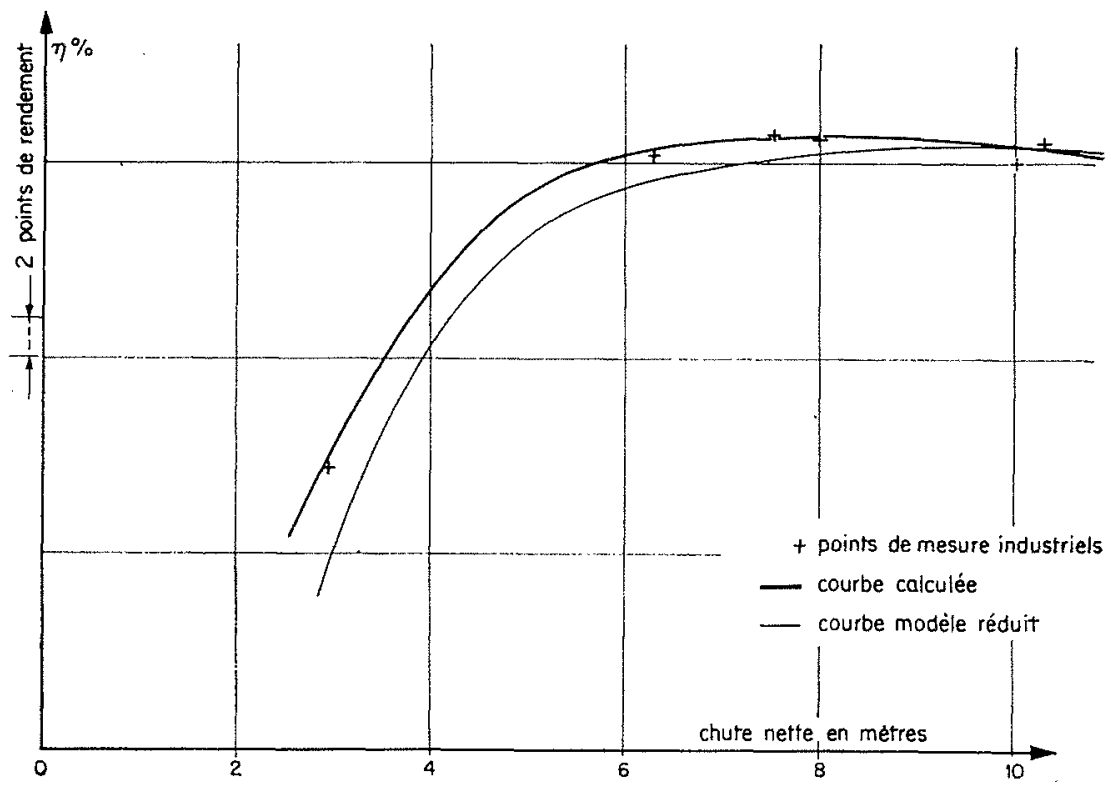

Fig. 4

Cambeyrac.

Turbinage inversé $i=12^{\circ} 5$. 
FIG. 5

Cambeyrac.
Turbinage inversé $i=20^{\circ}$.
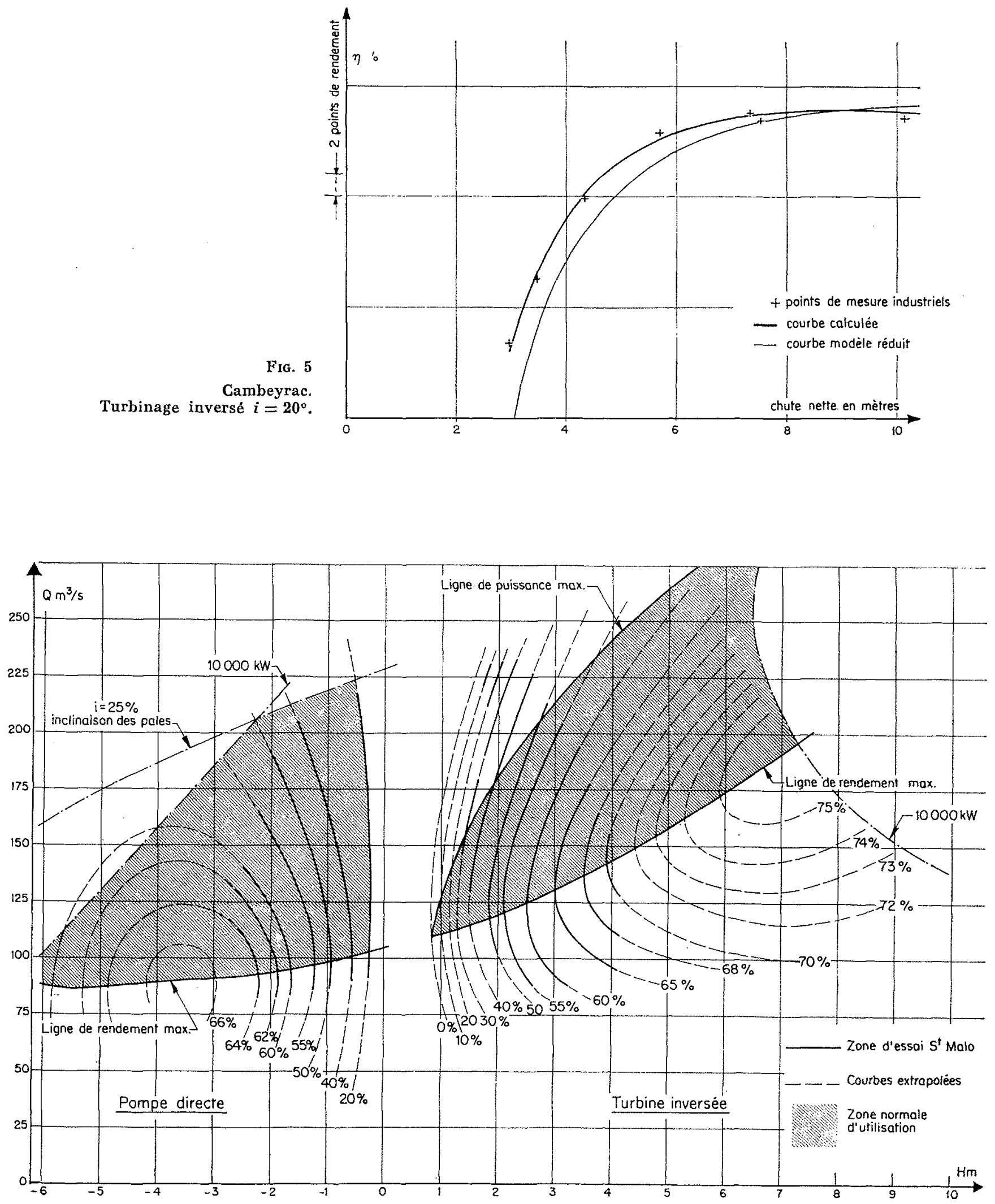

FIG. $5 a$

Saint-Malo. Diagramme topographique des rendements. 


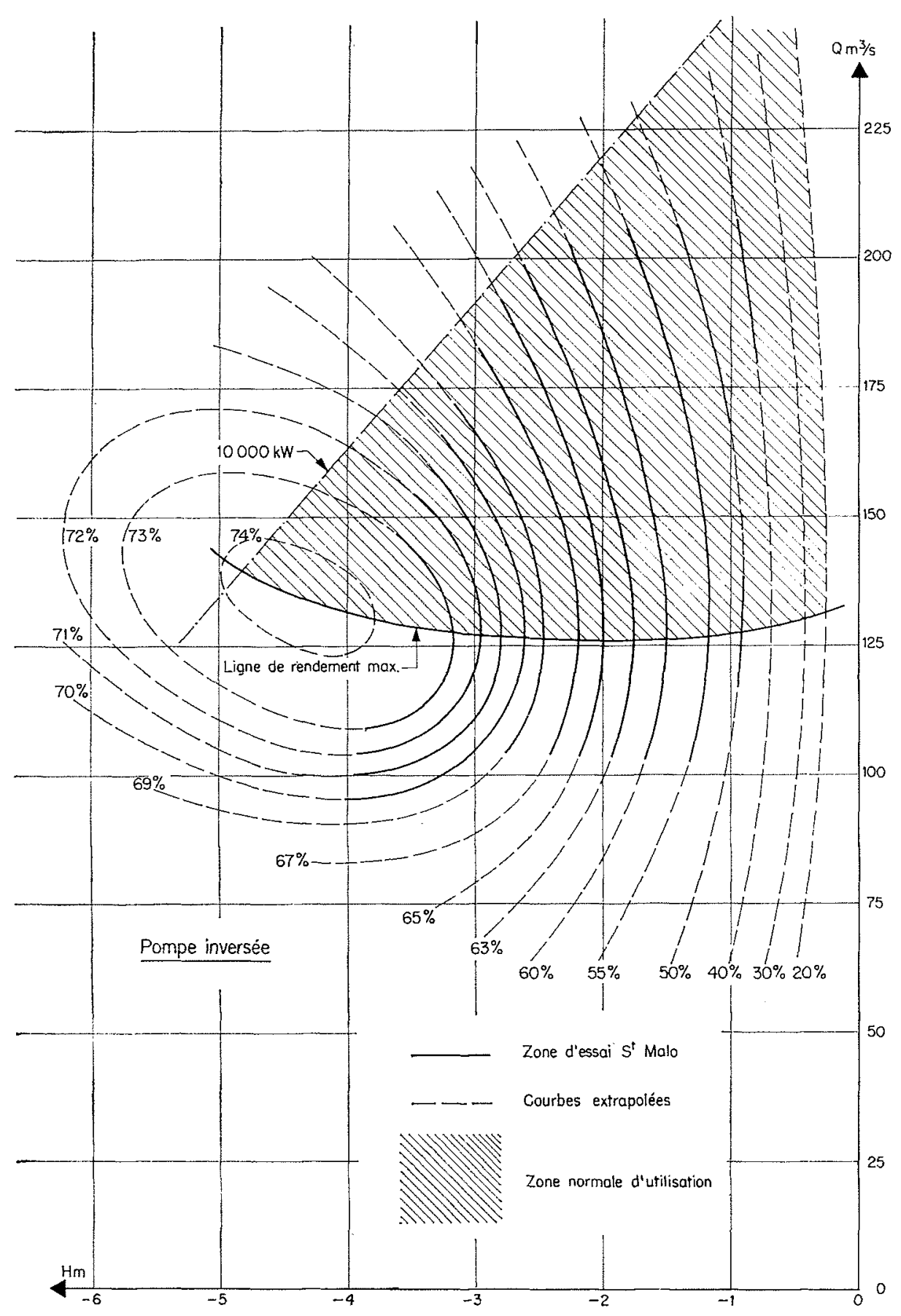

\section{II. - ESSAIS SAINT-MALO}

Fig. $5 b$

Saint-Malo.

Diagramme topographique du rendement.
A la demande de la Région d'Equipement Hydraulique $n^{\circ} 8$, nous avons exploité un modèle réduit de $500 \mathrm{~mm}$ de diamètre de roue, représentant à l'échelle $1 / 11,6$ le groupe marémoteur expérimental de Saint-Malo, y compris les ouvrages d'entonnement et de restitution depuis l'entrée de l'écluse côté bassin jusqu'à la sortie des vannes-papillons côté mer.
Le même effort de contrôle de la similitude gćométrique qu’à Cambeyrac a été poursuivi entre ce modèle et l'installation industrielle. De même, la définition des chutes et leurs emplacements de mesure ont été choisis de manière rigoureusement semblable.

Sur modèle, les caractéristiques complètes de fonctionnement ont été relevées dans les limites 
d'exploitation de ce même groupe à l'usine de la Rance, c'est-à-dire dans une gamme de chutes s'étendant de 0 à $10 \mathrm{~m}$.

Les essais industriels, dont on pouvait penser a priori qu'ils seraient difficiles et entachés d'imprécisions du fait des faibles chutes d'essais et de leur variation en cours de mesure, se sont au contraire révélés particulièrement homogènes, ainsi qu'a pu vous le montrer la communication de M. Dubois.

A la suite de ces essais forcément partiels du fait de la gamme d'utilisation réduite par rapport à la zone de fonctionnement Rance, il nous a été demandé de prévoir les caractéristiques réelles de ce même groupe implanté à l'usine de la Rance afin de déterminer l'effet d'échelle à attendre dans le reste de la gamme des chutes.

Bien que les essais industriels de Saint-Malo s'arrêtent à 4 mètres de chute, il nous a été possible, selon la méthode exposée précédemment, gnâce au nombre et à la valeur des points de mesures effectués dans la zone de 0 à $4 \mathrm{~m}$, de définir avec une excellente précision la pente des droites puissance-hauteur pour les différentes inclinaisons de pales réalisées, ainsi que le déca-

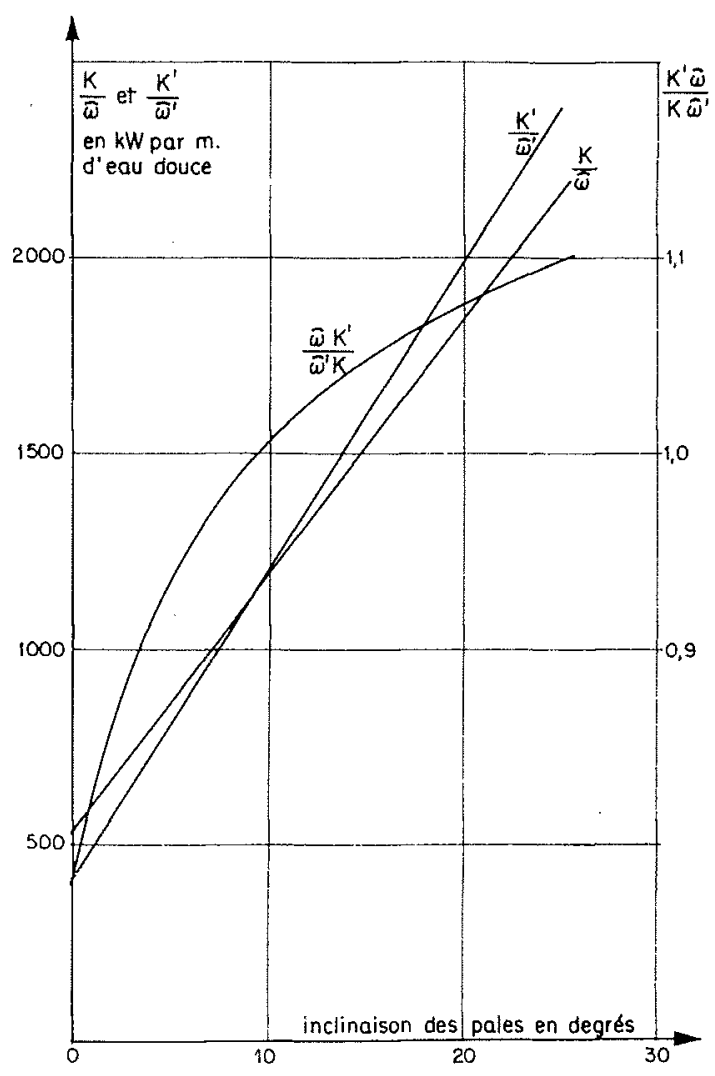

Fic. 6

Saint-Malo. Turbinage inversé. Pente des droites puissance-hauteur.

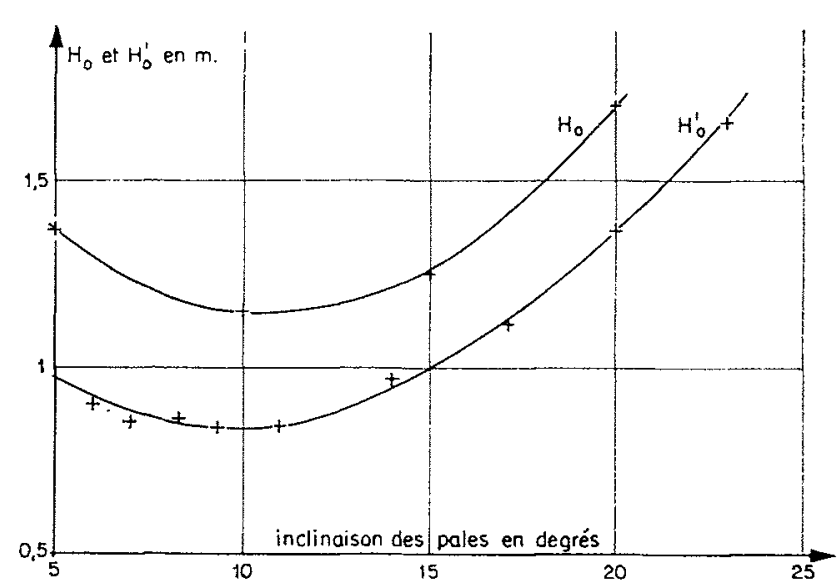

FIG. 7

Saint-Malo. Turbinage inversé.

Chutes minimales turbinables.

lage des chutes minimales turbinables et des courbes débit-hauteur.

Les courbes définissant la valeur des paramètres intervenant dans la relation (4) dans le cas de Saint-Malo, sont reproduites en annexe (fig. 6, 7, 8).

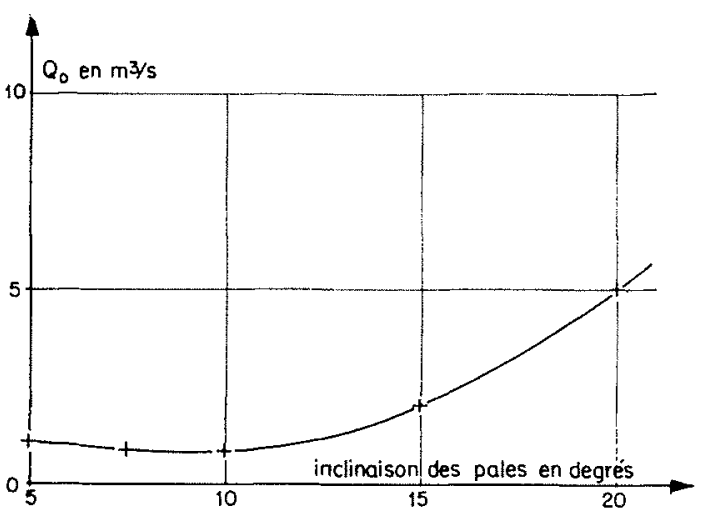

Frg. 8

Saint-Malo. Turbinage inversé. Décalage des courbes débit-hauteur.

L'application de la relation (4) aux résultats modèle relevés jusqu'à 10 mètres de chute, nous a permis, à partir des courbes rendement-hauteur mesurées, de calculer une colline industrielle tracée jusqu'à $9000 \mathrm{~kW}$, limite de puissance de l'alternateur Rance. L'ensemble de ces résultats figure en annexe (fig. 9 à 14).

Les puissances industrielles ont bien entendu été réduites de $3 \%$ environ pour tenir compte de la densité de l'eau de mer mesurée sur place et afin d'être directement comparables aux puissances modèle. 
Cette colline tient donc compte des résultats industriels mesurés aux essaís, des résultats obtenus sur le modèle et du décalage effectivement constaté dans la zone où la comparaison pouvait être faite directement.

Les bons résultats obtenus à Cambeyrac pour les fortes chutes nous autorisent à penser que l'erreur systématique qui peut être commise en fin de calcul n'excède pas un point de rendement par rapport au rendement qui pourrait y être effectivement mesuré.

Cette erreur est en tout cas bien inférieure à celle qui serait commise par la simple application d'une formule classique de transposition, dont nous avons pu d'ailleurs constater l'écart par rapport à la réalité dans la zone des essais industriels.

La même méthode a été appliquée pour les fonctionnements en pompe directe et inversée (fonctionnements à distributeur fixe) pour les- quels le calcul développé plus haut conduit à la relation suivante :

$$
\frac{\eta^{\prime}}{\eta}=\frac{K}{\mathrm{~K}^{\prime}} \frac{\omega^{\prime}}{\omega}\left(1-\mathrm{Q}_{0} / \mathrm{Q}\right)\left(1+\frac{\mathrm{H}_{0}^{\prime}-\mathrm{H}_{0}}{\mathrm{H}_{0}-\mathrm{H}_{0}^{\prime}}\right)
$$

avec les mêmes notations que pour la relation (4) et en ne définissant les rendements ainsi introduits que pour les hauteurs négatives (fig. 10).

Il serait également possible d'appliquer la même méthode à chacune des hélices partielles effectuées lors de la réalisation des essais en turbinage direct. Malheureusement le faible nombre d'essais qu'il a été possible de réaliser sur place en turbine directe n'a permis que de déterminer la colline conjuguée dans la zone des faibles chutes. En effectuant cependant quelques essais déconjugués pour ces chutes, mais correspondant $\grave{a}$ une conjugaison pour les fortes chutes de la Rance, la méthode précédente permet de calculer un rendement conjugué

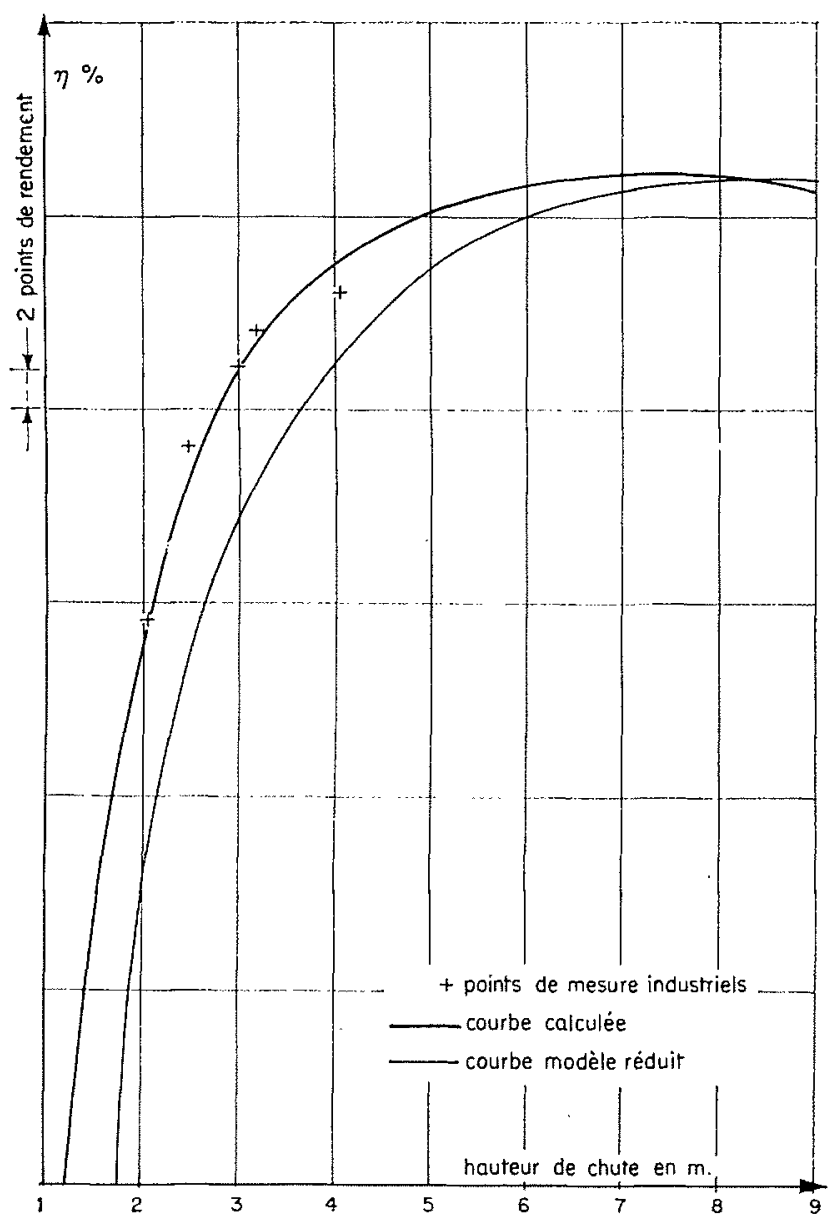

Fig. 9

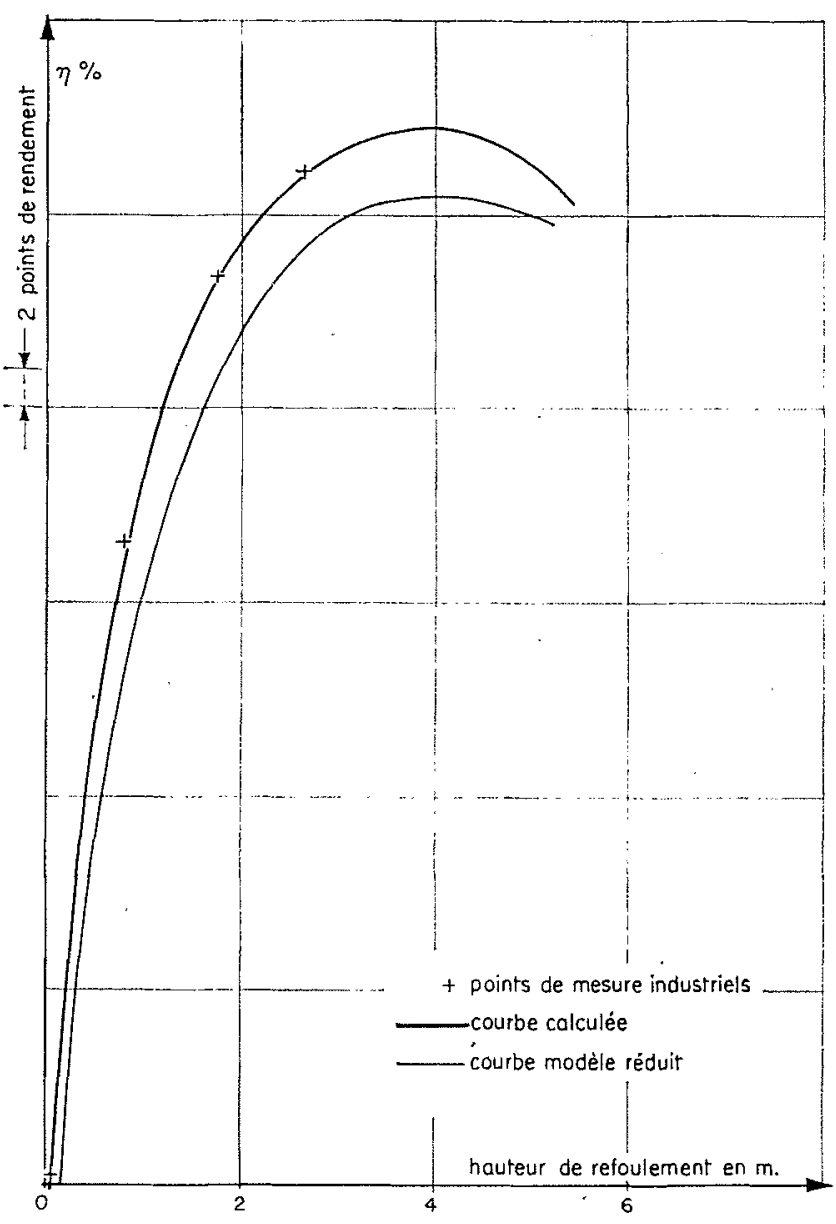

Fig. 10

Saint-Malo. Pompage direct $i=14^{\circ}$. 


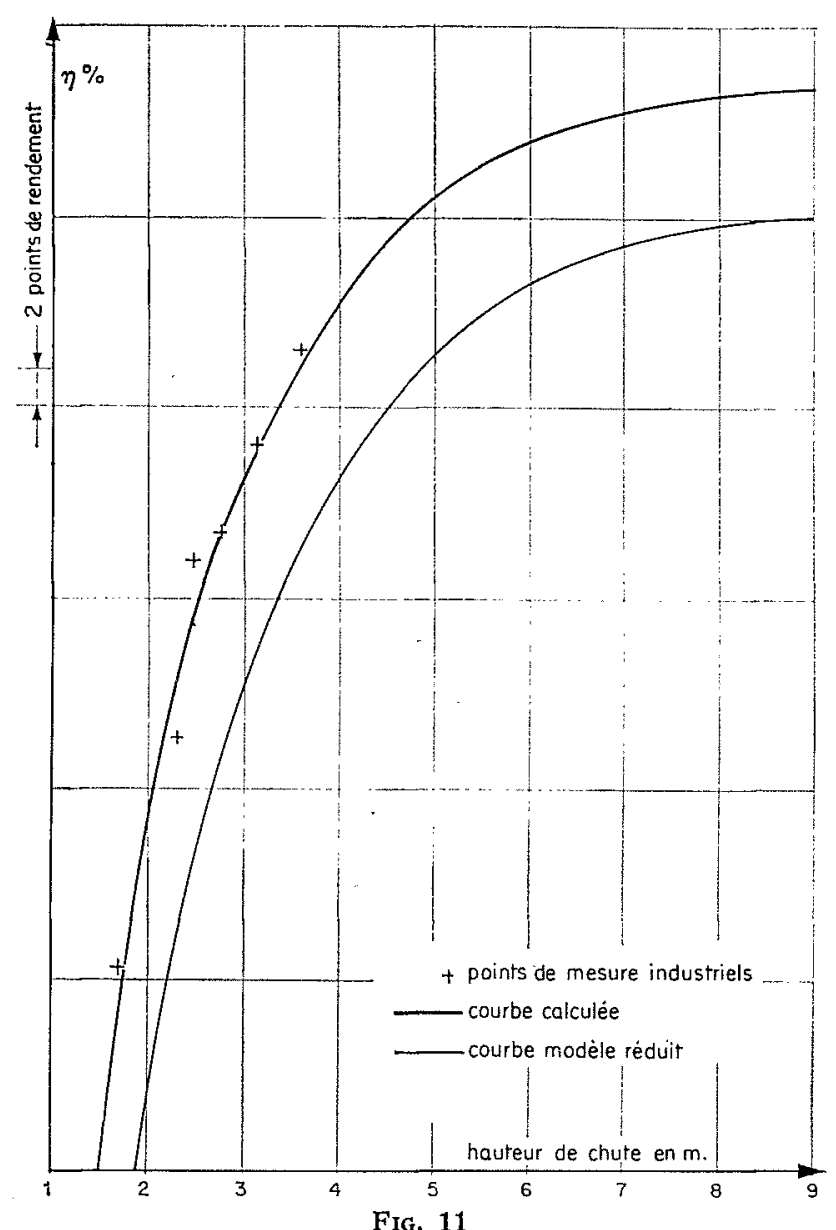

Saint-Malo. Turbinage inverse $i=17^{\circ} 2$.

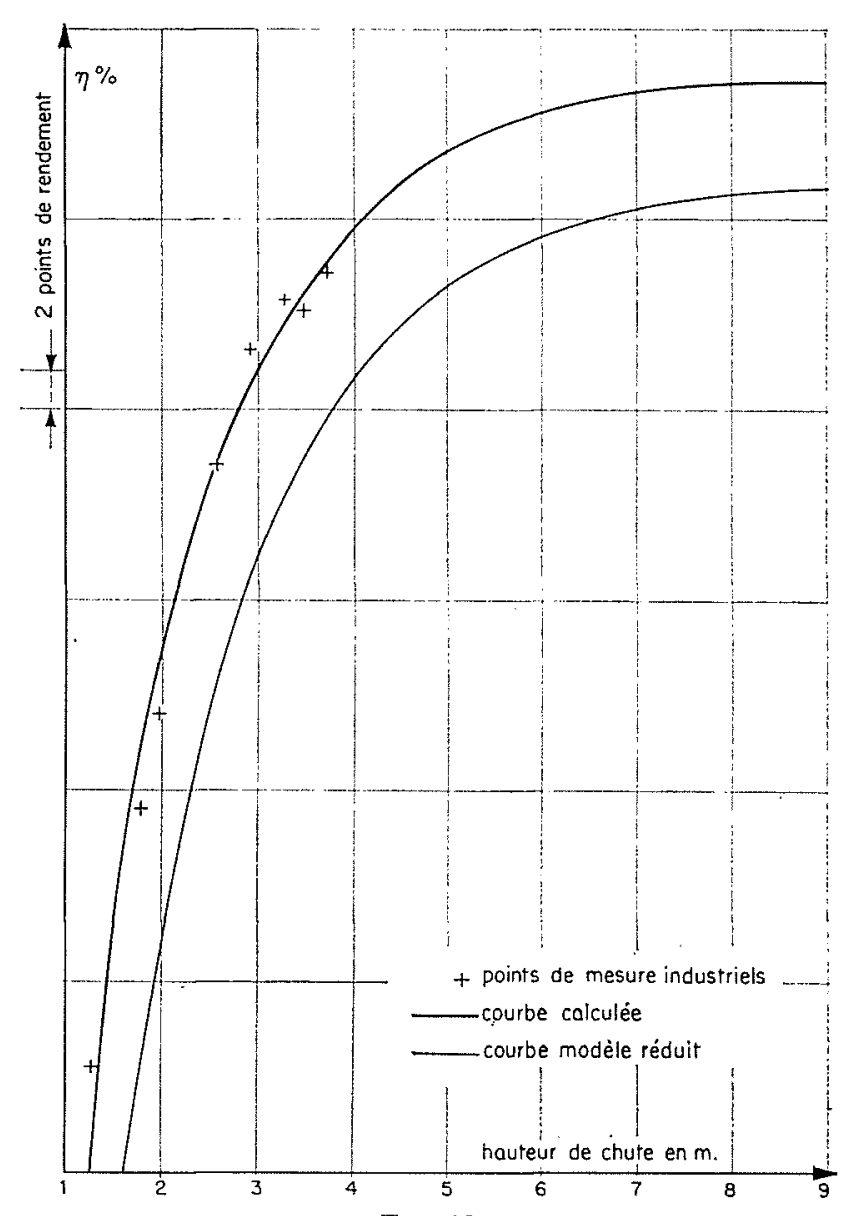

FIG. 12

Saint-Malo. Turbinage inversé $i=14^{\circ} 2$.

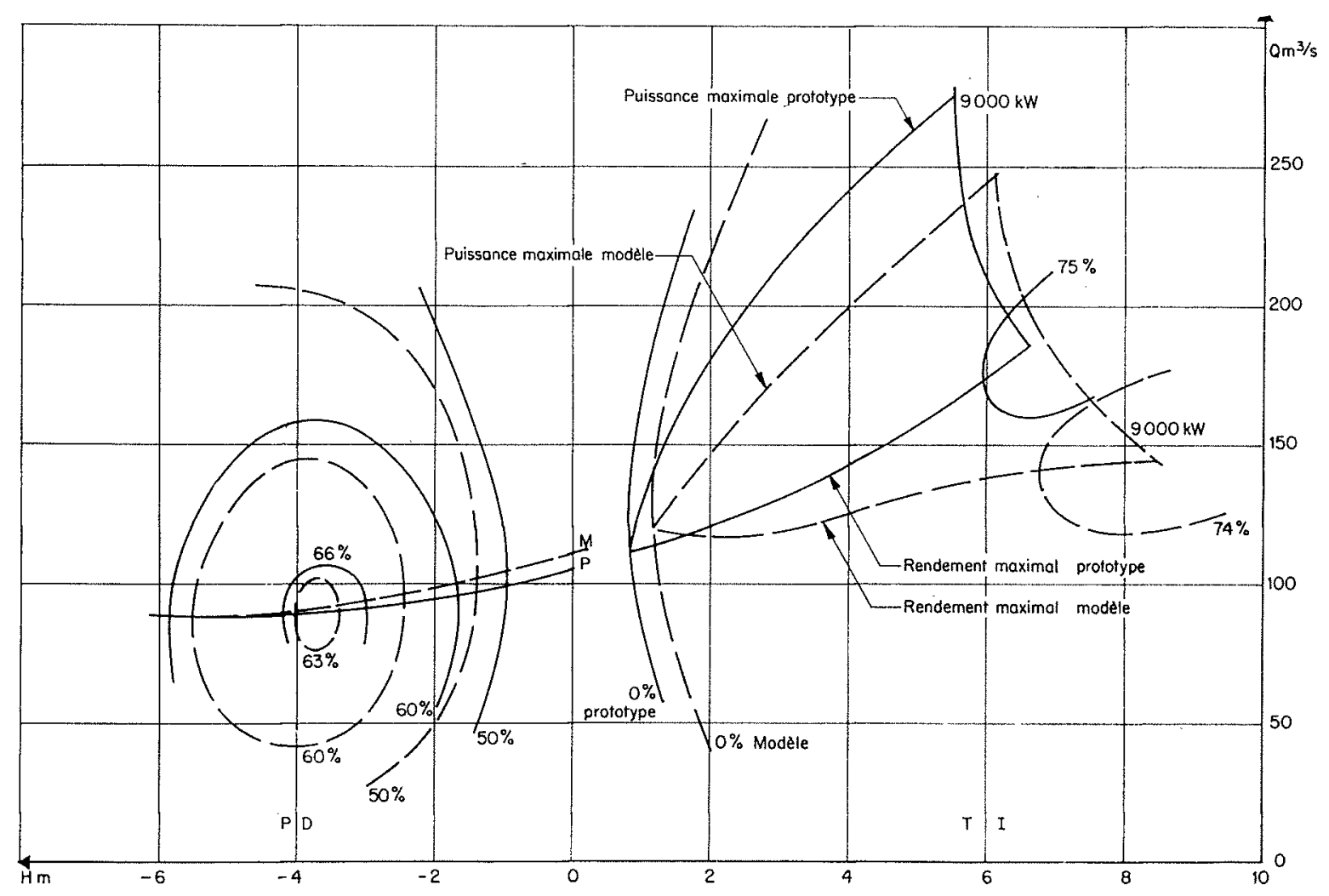

FIG. 13

Saint-Malo. Comparaison entre les essais modèle et l'extrapolation des essais industriels. Pompe directe. Turbine inversée. 
Fig. 14

Saint-Malo. Comparaison entre les essais modèle et l'extrapolation des essais industriels. Pompe inversée.

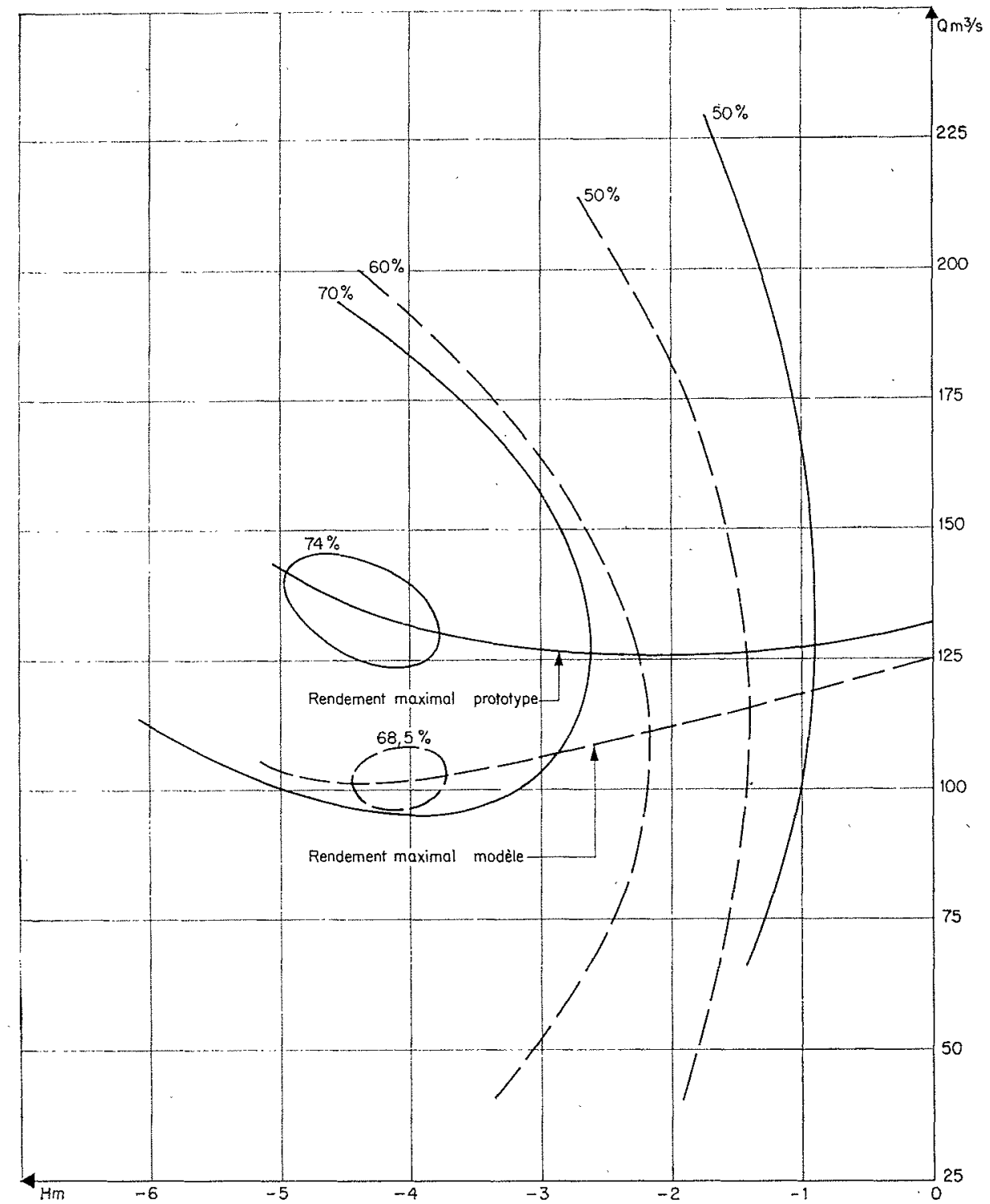

dans la zone de $9000 \mathrm{~kW}$, précisant l'effet d'échelle à attendre dans cette région.

La comparaison des valeurs obtenues sur modèle et sur le prototype est donnée dans les collines 15 et 16 .

\section{*}

L'application directe du calcul présenté a done permis, sous le couvert des bons recoupements effectués sur la machine de Cambeyrac et, moyennant les précautions énumérées, grâce à la confiance qu'on peut accorder aux essais industriels, de préciser la valeur absolue des performances à attendre d'une machine type SaintMalo installée dans le même cadre, mais avec les gammes de fonctionnement Rance.

Il resterait alors, d'un point de vue plus théorique, à essayer de rattacher les trois paramètres intervenant dans ce calcul aux caractéristiques hydrauliques de ces machines. Ceci permettrait de transformer cette formule de passage en une véritable formule de transposition ne nécessitant plus l'exécution des essais industriels. De nouveaux essais effectués sur des machines similaires doivent pouvoir apporter à ce sujet des renseignements utiles. 


\section{DIS C U S I I N}

président : M. GIBRat

M. le Président remercie les deux conférenciers et ouvre la discussion, aussi bien sur les groupes de Cambeyrac et de Saint-Malo et sur leur rendement que sur les méthodes de transposition qui ont été adoptées.

M. AURoy demande s'il y a une explication hydraulique aux fluctuations de pression observées par M. DvBors dans le fonctionnement en orifice du groupe de Saint-Malo aux faibles ouvertures du distributeur, fluctuations dont la période, à peu près constante, était -sensiblement le triple de celle correspondant à la vitesse de rotation de la roue et dont l'amplitude allait en diminuant au fur et à mesure que l'ouverture du distributeur augmentait.

M. Dubors croit que ces fluctuations sont dues aux phénomènes du décollement tournant, qu'on observe de façon assez classique sur les compresseurs et les pompes, mais qu'on n'avait pas encore observé, du moins à sa connaissance, sur les turbines. C'est un décollement qui se localise entre deux pales de la machine et passe d'un couple de pales à l'autre avec une vitesse qui est différente de celle de la machine, mais dans un rapport que $M$. Dubors ne peut préciser. Ces fluctuations de pression semblent être un phénomène très local, décelé seulement par des prises de pression très voisines des pales, et non pas une oscillation de l'ensemble du conduit hydraulique, laquelle apparaîtrait - mais n'apparait pas en fait - dans les mesures de la chute et de la vitesse de rotation de la machine.

M. Le Menestrel ajoute que ces fluctuations se produisent parce que le fonctionnement en orifice est très «sous-conjugué» par rapport à l'inclinaison des pales : elles disparaissent en conjugaison et peuvent même réapparaître en «sur-conjugaison».

M. HENRY se réfèrè à une formule très intéressante exposée par M. Chevalier pour déterminer les rendements pour des zones non explorées à partir du modèle, d'une part, et à partir des zones résultats du prototype, d'autre part: les résultats donnés par cette formule diffèrent-ils notablement de ce que donnent les formules de transposition classiques?

M. Chevalien répond que les écarts les plus satisfaisants entre les résultats des deux formules se situent à $\pm 2 \%$, mais que les résultats ne sont pas comparables, car les formules de transposition classiques ne s'appliquent pas aux machines axiales à un seul réglage, c'est-à-dire avec distributeur fixe.

M. Lieber demande à M. Chevalirer où en sont les recherches poursuivies par les Services d'E.d.F., dans le cadre des essais de Cambeyrac et de Chatou, pour établir une formule de transposition plus générale que celles dont il a été fait état par M. Chevalier et qui s'appliquent à des cas particuliers.

M. Chevalier répond qu'il ne s'agit pas de trouver une formule plus générale, mais qu'il s'agit, dans ce cas bien particulier de la machine à un seul réglage, d'essayer de transformer ce qui a été trouvé en une véritable formule de transposition; c'est-à-dire d'y faire apparaître le nombre de Reynolds.
M. Chevrier fait une remarque d'un ordre tout à fait général :

«L'originalité et la nouveauté des projets d'usines marémotrices ont permis de pousser l'expérimentation et les essais à un niveau bien supérieur à celui dont pouvaient disposer les utilisateurs de l'énergie hydraulique classique, comme nous venons de le constater par les intéressants exposés des rapporteurs.

«Il faut se féliciter, en particulier, que la confrontation ait pu être poussée aussi loin entre un modèle réduit de turbine et la machine en vraie grandeur. S'agissant de machines classiques Francis ou Kaplan, les exploitants ont été souvent surpris de différences très importantes entre les résultats qu'ils constataient et ceux qu'ils auraient pu espérer d'après les essais sur des modèles réduits. Je pense, en particulier, aux mesures et essais de M. Fontaine dans ses aspirateurs de turbines de basse et moyenne chute et qui ont révélé des phénomènes que l'étude des modèles rẻduits n'avait pas décelés.

« Il serait intéressant de savoir si les résultats d'essaís qui viennent de nous être exposés peuvent faire espérer une meilleure connaissance des lois de similitude entre modèles réduits et turbines vraie grandeur, pour les modèles classiques de turbines utilisées en hydaulique fluviale.»

M. WhLL indique que, dans le cadre des mesures qui permettent effectivement de mieux connaître le comportement hydraulique des turbines, et en particulier d'autoriser des comparaisons entre bulbes industriels et modèles, il est intéressant de rappeler les résultats très substantiels obtenus par M. CaMpMas au moyen de tubes de Pitot sur le groupe bulbe de Beaumont-Monteux, résultats qu'il a eu l'occasion d'exposer ici-même et qui ont fait l'objet d'un récent article dans $L a$ Houille Blanche. A la suite de ces essais, il a été prévu d'équiper quelques groupes de la Rance des dispositifs permettant la mise en place de tubes de Pitot, de part et d'autre de la roue et à $90^{\circ}$ les uns des autres.

M. Hug rappelle que les formules de transposition ne sont utilisables que dans la limite même des conditions où elles ont été établies. Or, jusqu'à présent, ces formules résultent rarement, pour ne pas dire jamais, de la comparaison de résultats obtenus sur un modèle avec les résultats des mesures effectuées sur la machine industrielle, elle-même construite d'après ce modèle. Cette discordance $a$ priori rend très difficile et très aléatoire le critère de leur validité.

Le Département Essais d'E.d.F. se propose de choisir un certain nombre de cas particuliers correspondant à des couples de modèles et de machines industrielles conformes et où il est possible d'appliquer une méthode de travail aussi analytique et complète que possible, de manière à ne faire entrer dans la formule que les effets d'échelle, excluant autant que possible les écarts de géométrie et les erreurs systématiques dans les mesures.

M. FerRY fait observer que le rendement de la machine industrielle, dans les zones où l'on manque de mestures, est calculé en partant d'estimations de la puissance et du débit, en fonction de $\mathrm{H}$ et du paramètre inclinaison des pales. L'estimation de la puis- 
sance se fonde sur la possibilité d'une représentation de celle-ci par une famille de droites; celle du débit, sur une loi de correspondance simple avec le débit du modèle. Mais pour obtenir le rendement à $1 \%$ près, il est nécessaire que les estimations précédentes soient elles-mêmes précises à mieux que $1 \%$. Dans ces conditions, on peut se demander si cette précision, possible lorsqu'il s'agit d'interpoler dans les limites de la zone couverte par les essais industriels, n'est pas difficile à atteindre lorsqu'il s'agit d'extrapolcr à l'extérieur de cette zone.

M. Chevalier répond que les courbes présentées dans ce rapport sont obtenues pour la machine de Cambeyrac dans toute la zone de son utilisation. On a donc pu vérifier que le tracé de la droite $W=f(h)$ obtenu à partir de ces résultats ne différait pas de plus de $1 \%$ du tracé que l'on aurait pu obtenir en ne connaissant que les points relatifs à une zone de fonctionnement pIns réduite. L'écart de $1 \%$ est en fait dù à la dispersion des points de mesure plutôt qu'à la non-linéarité de la courbe.

M. Hug confirme et résume en indiquant : « Nous avons mesuré à Cambeyrac un $\Delta Q$ et un $\Delta W$, ou, plus généralement, certains caractères constants dans toute la gamme de fonctionnement et nous disons que, dans une machine analogue et dans la gamme de fonctionnement analogue, nous mesurons localement le $\Delta Q$ et le $\Delta W$ et nous pensons que ces caractères restent constants dans toute la gamme de fonctionnement, comme nous avons pu le vérifier sur la machine analogue de Cambeyrac. En fait, ceci n'est pas une extrapolation. 》

M. Chevalier précise que l'on aurait rencontré des difficultés plus importantes si l'on avait été amené à faire le même travail sur la turbine directe: là, effectivement, il aurait fallu se placer loin de la conjugaison et en préjuger à l'avance.

M. le Président interprète que MM. Hug et Chevalien revendiquent l'application d'une méthode qui s'est vérifiée expérimentalement parfaite à Cambeyrac à un sujet qui leur apparait assez voisin, mais non à une turbine classique (Pelton, Francis ou Kaplan verticale).

M. le Prësident demande si des méthodes analogues ne pourraient pas être envisagées pour déterminer les rendements en vraie grandeur de turbines ou de soufflantes à air ou à gaz, à partir de mesures faites sur un modèle. Ces problèmes se posent dans l'industrie nucléaire, où on ne possède pas d'installation suffisante pour faire des essais en vraie grandeur à tous les régimes.

M. Dubors répond que des essais sur place en vraie grandeur ont été effectués récemment sur les ventilateurs de tirage de l'usine de Champagne-sur-Oise, le débit étant mesuré par un diaphragme installé dans une gaine spéciale pour essais. D'une façon plus générale, la mesure du débit (la seule qui pose un problème) peut être réalisée pour des gaz par exploration du champ des vitesses dans des conditions analogues à celles des essais de turbines hydrauliques.

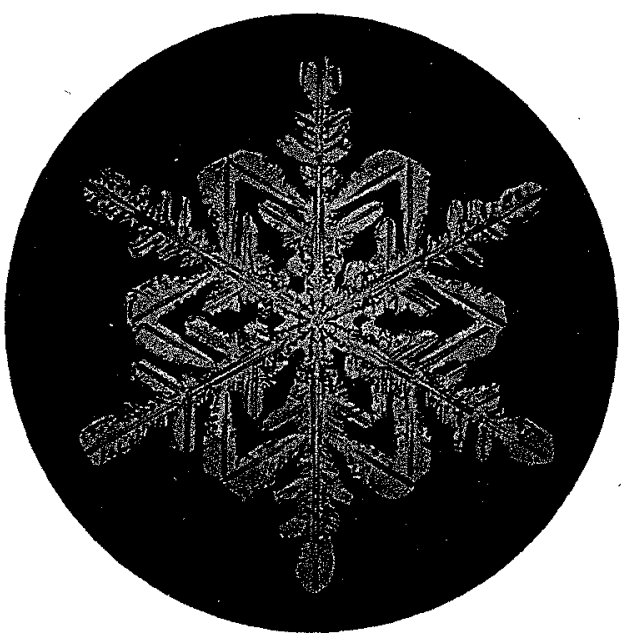

